\title{
Sharp Integral Inequalities Involving High-Order Partial Derivatives
}

\author{
C.-J Zhao' ${ }^{1}$ and W.-S Cheung ${ }^{2}$ \\ ${ }^{1}$ Department of Information and Mathematics Sciences, College of Science, China Jiliang University, \\ Hangzhou 310018, China \\ ${ }^{2}$ Department of Mathematics, The University of Hong Kong, Pokfulam Road, Hong Kong
}

Correspondence should be addressed to C.-J Zhao, chjzhao@163.com

Received 28 November 2007; Accepted 10 April 2008

Recommended by Peter Pang

The main purpose of the present paper is to establish some new sharp integral inequalities involving higher-order partial derivatives. Our results in special cases yield some of the recent results on Agarwal, Wirtinger, Poincaré, Pachpatte, Smith, and Stredulinsky's inequalities and provide some new estimates on such types of inequalities.

Copyright ( 12008 C.-J Zhao and W.-S Cheung. This is an open access article distributed under the Creative Commons Attribution License, which permits unrestricted use, distribution, and reproduction in any medium, provided the original work is properly cited.

\section{Introduction}

Inequalities involving functions of $n$ independent variables, their partial derivatives, integrals play a fundamental role in establishing the existence and uniqueness of initial and boundary value problems for ordinary and partial differential equations as well as difference equations [1-10]. Especially, in view of wider applications, inequalities due to Agarwal, Opial, Pachpatte, Wirtinger, Poincaré and et al. have been generalized and sharpened from the very day of their discover. As a matter of fact, these now have become research topic in their own right [11-14]. In the present paper, we will use the same method of Agarwal and Sheng [15], establish some new estimates on these types of inequalities involving higher-order partial derivatives. We further generalize these inequalities which lead to result sharper than those currently available. An important characteristic of our results is that the constant in the inequalities are explicit.

\section{Main results}

Let $R$ be the set of real numbers and $R^{n}$ the $n$-dimensional Euclidean space. Let $E, E^{\prime}$ be a bounded domain in $R^{n}$ defined by $E \times E^{\prime}=\prod_{i=1}^{n}\left[a_{i}, b_{i}\right] \times\left[c_{i}, d_{i}\right], i=1, \ldots, n$. For $x_{i}, y_{i} \in R, i=1, \ldots, n,(x, y)=\left(x_{1}, \ldots, x_{n}, y_{1}, \ldots, y_{n}\right)$ is a variable point in $E \times E^{\prime}$ and 
$d x d y=d x_{1} \cdots d x_{n} d y_{1} \cdots d y_{n}$. For any continuous real-valued function $u(x, y)$ defined on $E \times E^{\prime}$, we denote by $\int_{E} \int_{E^{\prime}} u(x, y) d x d y$ the $2 n$-fold integral

$$
\int_{a_{1}}^{b_{1}} \ldots \int_{a_{n}}^{b_{n}} \int_{c_{1}}^{d_{1}} \cdots \int_{c_{n}}^{d_{n}} u\left(x_{1}, \ldots, x_{n}, y_{1}, \ldots, y_{n}\right) d x_{1} \cdots d x_{n} d y_{1} \cdots d y_{n}
$$

and for any $(x, y) \in E \times E^{\prime}, \int_{E(x)} \int_{E^{\prime}(x)} u(s, t) d s d t$ is the $2 n$-fold integral

$$
\int_{a_{1}}^{x_{1}} \cdots \int_{a_{n}}^{x_{n}} \int_{c_{1}}^{y_{1}} \cdots \int_{c_{n}}^{y_{n}} u\left(s_{1}, \ldots, s_{n}, t_{1}, \ldots, t_{n}\right) d x_{1} \cdots d s_{n} d t_{1} \cdots d t_{n} .
$$

We represent by $F\left(E \times E^{\prime}\right)$ the class of continuous functions $u(x, y): E \times E^{\prime} \rightarrow R$ for which $D^{2 n} u(x, y)=D_{1} \cdots D_{2 n} u(x, y)$, where

$$
D_{1}=\frac{\partial}{\partial x_{1}}, \ldots, D_{n}=\frac{\partial}{\partial x_{n}}, D_{n+1}=\frac{\partial}{\partial y_{1}}, \ldots, D_{2 n}=\frac{\partial}{\partial y_{n}}
$$

exists and that for each $i, 1 \leq i \leq n$,

$$
\left.u(x, y)\right|_{x_{i}=a_{i}}=0,\left.\quad u(x, y)\right|_{y_{i}=c_{i}}=0,\left.\quad u(x, y)\right|_{x_{i}=b_{i}}=0,\left.\quad u(x, y)\right|_{y_{i}=d_{i}}=0, \quad(i=1, \ldots, n)
$$

the class $F\left(E \times E^{\prime}\right)$ is denoted as $G\left(E \times E^{\prime}\right)$.

Theorem 2.1. Let $\mu \geq 0, \lambda \geq 1$ be given real numbers, and let $p(x, y) \geq 0,(x, y) \in E \times E^{\prime}$ be a continuous function. Further, let $u(x, y) \in G\left(E \times E^{\prime}\right)$. Then, the following inequality holds

$$
\begin{aligned}
& \int_{E} \int_{E^{\prime}} p(x, y)|u(x, y)|^{\mu} d x d y \\
& \quad \leq \int_{E} \int_{E^{\prime}} p(x, y) q(x, y, \lambda, \mu) d x d y\left(\int_{E} \int_{E^{\prime}}\left|D^{2 n} u(x, y)\right|^{\lambda} d x d y\right)^{\mu / \lambda}
\end{aligned}
$$

where

$$
q(x, y, \lambda, \mu)=\left(\frac{1}{2^{n+1}} \prod_{i=1}^{n}\left[\left(x_{i}-a_{i}\right)\left(b_{i}-x_{i}\right)\left(y_{i}-c_{i}\right)\left(d_{i}-y_{i}\right)\right]^{(\lambda-1) / 2}\right)^{\mu / \lambda}
$$

Proof. For the set $\{1, \ldots, n\}$, let $\pi=A \cup B, \pi^{\prime}=A^{\prime} \cup B^{\prime}$ be partitions, where $A=\left(j_{1}, \ldots, j_{k}\right), B=$ $\left(j_{k+1}, \ldots, j_{n}\right), A^{\prime}=\left(i_{1}, \ldots, i_{k}\right)$, and $B^{\prime}=\left(i_{k+1}, \ldots, i_{n}\right)$ are such that card $A=\operatorname{card} A^{\prime}=k$ and card $B=\operatorname{card} B^{\prime}=n-k, 0 \leq k \leq n$. It is clear that there are $2^{n+1}$ such partitions. The set of all such partitions we will denote as $Z$ and $Z^{\prime}$, respectively. For fixed partition $\pi, \pi^{\prime}$ and $x \in E, y \in E^{\prime}$, we define

$$
\int_{E_{\pi}(x)} \int_{E_{\pi^{\prime}}^{\prime}(y)} u(s, t) d s d t=\int_{A(x)} \int_{B(x)} \int_{A^{\prime}(y)} \int_{B^{\prime}(y)} u(s, t) d s d t
$$


where $\int_{A(x)^{\prime}} \int_{A^{\prime}(y)}$ denote the $k$-fold integral, $\int_{B(x)^{\prime}} \int_{B^{\prime}(y)}$ represent the $(n-k)$-fold integral. Thus from the assumptions it is clear that for each $\pi \in Z, \pi^{\prime} \in Z^{\prime}$

$$
|u(x, y)| \leq \int_{E_{\pi}(x)} \int_{E_{\pi^{\prime}}^{\prime}(y)}\left|D^{2 n} u(s, t)\right| d s d t .
$$

In view of Hölder integral inequality, we have

$$
\begin{aligned}
|u(x, y)| \leq & \left(\prod_{i \in A}\left(x_{i}-a_{i}\right) \prod_{i \in B}\left(b_{i}-x_{i}\right) \prod_{i \in A^{\prime}}\left(y_{i}-c_{i}\right) \prod_{i \in B^{\prime}}\left(d_{i}-y_{i}\right)\right)^{(\lambda-1) / \lambda} \\
& \times\left(\int_{E_{\pi}(x)} \int_{E_{\pi^{\prime}}^{\prime}(y)}\left|D^{2 n} u(s, t)\right|^{\lambda} d s d t\right)^{1 / \lambda} .
\end{aligned}
$$

A multiplication of these $2^{n+1}$ inequalities and an application of the Arithmetic-Geometric mean inequality give

$$
\begin{aligned}
|u(x, y)|^{\mu} \leq & \left(\prod_{i=1}^{n}\left[\left(x_{i}-a_{i}\right)\left(b_{i}-x_{i}\right)\left(y_{i}-c_{i}\right)\left(d_{i}-y_{i}\right)\right]^{(\lambda-1) / 2}\right)^{\mu / \lambda} \\
& \times\left(\prod_{\pi \in Z, \pi^{\prime} \in Z^{\prime}}\left(\int_{E_{\pi}(x)} \int_{E_{\pi^{\prime}}^{\prime}(y)}\left|D^{2 n} u(s, t)\right|^{\lambda} d s d t\right)^{1 / 2^{n+1}}\right)^{\mu / \lambda} \\
\leq & \left(\frac{1}{2^{n+1}} \prod_{i=1}^{n}\left[\left(x_{i}-a_{i}\right)\left(b_{i}-x_{i}\right)\left(y_{i}-c_{i}\right)\left(d_{i}-y_{i}\right)\right]^{(\lambda-1) / 2}\right)^{\mu / \lambda} \\
& \times\left(\sum_{\pi \in Z, \pi^{\prime} \in Z^{\prime}} \int_{E_{\pi}(x)} \int_{E_{\pi^{\prime}}^{\prime}(y)}\left|D^{2 n} u(s, t)\right|^{\lambda} d s d t\right)^{\mu / \lambda} \\
= & q(x, y, \lambda, \mu)\left(\int_{E} \int_{E^{\prime}}\left|D^{2 n} u(s, t)\right|^{\lambda} d s d t\right)^{\mu / \lambda} .
\end{aligned}
$$

Now, multiplying both the sides of $(2.10)$ by $p(x, y)$ and integrating the resulting inequality on $E \times E^{\prime}$, we have

$$
\int_{E} \int_{E^{\prime}} p(x, y)|u(x, y)|^{\mu} d x d y \leq \int_{E} \int_{E^{\prime}} p(x, y) q(x, y, \lambda, \mu) d x d y\left(\int_{E} \int_{E^{\prime}}\left|D^{2 n} u(s, t)\right|^{\lambda} d s d t\right)^{\mu / \lambda}
$$

where

$$
q(x, y, \lambda, \mu)=\left(\frac{1}{2^{n+1}} \prod_{i=1}^{n}\left[\left(x_{i}-a_{i}\right)\left(b_{i}-x_{i}\right)\left(y_{i}-c_{i}\right)\left(d_{i}-y_{i}\right)\right]^{(\lambda-1) / 2}\right)^{\mu / \lambda}
$$

Remark 2.2. Taking for $p(x, y)=1$ in $(2.5),(2.5)$ reduces to

where

$$
\int_{E} \int_{E^{\prime}}|u(x, y)|^{\mu} d x d y \leq K_{0}^{\prime}\left(\int_{E} \int_{E^{\prime}}\left|D^{2 n} u(x, y)\right|^{\lambda} d x d y\right)^{\mu / \lambda}
$$

$$
K_{0}^{\prime}=\left(\left(\frac{1}{2}\right)^{\mu / \lambda} B^{2}\left(1+\frac{\mu}{2}-\frac{\mu}{2 \lambda}, 1+\frac{\mu}{2}-\frac{\mu}{2 \lambda}\right)\right)^{n} \prod_{i=1}^{n}\left[\left(b_{i}-a_{i}\right)\left(d_{i}-c_{i}\right)\right]^{1+\mu-\mu / \lambda},
$$

and $B$ is the Beta function. 
Taking for $\lambda=\mu=2$ in (2.13) reduces to

$$
\int_{E} \int_{E^{\prime}}|u(x, y)|^{2} d x d y \leq\left(\frac{\pi^{2}}{8}\right)^{n} M^{\prime 2}\left(\int_{E} \int_{E^{\prime}}\left|D^{2 n} u(x, y)\right|^{2} d x d y\right),
$$

where

$$
M^{\prime}=\prod_{i=1}^{n} \frac{\left(b_{i}-a_{i}\right)\left(d_{i}-c_{i}\right)}{4} .
$$

Let $u(x, y)$ reduce to $u(x)$ in (2.15) and with suitable modifications, then (2.15) becomes the following two Wirting type inequalities:

$$
\int_{E}|u(x)|^{2} d x \leq\left(\frac{\pi}{4}\right)^{n} M^{2}\left(\int_{E}\left|D^{n} u(x)\right|^{2} d x\right)
$$

where

$$
M=\prod_{i=1}^{n} \frac{\left(b_{i}-a_{i}\right)}{2}
$$

Similarly

$$
\int_{E}|u(x)|^{4} d x \leq\left(\frac{3 \pi}{16}\right)^{n} M^{4}\left(\int_{E}\left|D^{n} u(x)\right|^{4} d x\right),
$$

where $M$ is as in (2.17).

For $n=2$, the inequalities (2.17) and (2.19) have been obtained by Smith and Stredulinsky [16], however, with the right-hand sides, respectively, multiplies $(4 / \pi)^{2}$ and $(16 / 3 \pi)^{4}$. Hence, it is clear that inequalities (2.17) and (2.19) are more strengthed.

Remark 2.3. Let $u(x, y)$ reduce to $u(x)$ in (2.5) and with suitable modifications, then (2.5) becomes the following result:

$$
\int_{E} p(x)|u(x)|^{\mu} d x \leq \int_{E} p(x) q(x, \lambda, \mu) d x\left(\int_{E}\left|D^{n} u(x)\right|^{\lambda} d x\right)^{\mu / \lambda},
$$

where

$$
q(x, \lambda, \mu)=\left(\frac{1}{2^{n}} \prod_{i=1}^{n}\left[\left(x-a_{i}\right)\left(b_{i}-x_{i}\right)\right]^{(\lambda-1) / 2}\right)^{\mu / \lambda} .
$$

This is just a new result which was given by Agarwal and Sheng [15].

Theorem 2.4. Let $p(x, y) \geq 0,(x, y) \in E \times E^{\prime}$ be a continuous function. Further, let for $k=$ $1, \ldots, r, \mu_{k} \geq 0, \lambda_{k} \geq 1$, be given real numbers such that $\sum_{k=1}^{r}\left(\mu_{k} / \lambda_{k}\right)=1$, and $u_{k}(x, y) \in G\left(E \times E^{\prime}\right)$. Then the following inequality holds

$$
\begin{aligned}
& \int_{E} \int_{E^{\prime}} p(x, y) \prod_{k=1}^{r}\left|u_{k}(x, y)\right|^{\mu_{k}} d x d y \\
& \quad \leq \int_{E} \int_{E^{\prime}} p(x, y) \prod_{k=1}^{r} q\left(x, y, \lambda_{k}, \mu_{k}\right) d x d y \sum_{k=1}^{r} \frac{\mu_{k}}{\lambda_{k}} \int_{E} \int_{E^{\prime}}\left|D^{2 n} u_{k}(x, y)\right|^{\lambda_{k}} d x d y .
\end{aligned}
$$


Proof. Setting $\mu=\mu_{k}, \lambda=\lambda_{k}$ and $u(x, y)=u_{k}(x, y), 1 \leq k \leq r$ in (2.10), multiplying the $r$ inequalities, and applying the extended Arithmetic-Geometric means inequality,

$$
\prod_{k=1}^{r} a_{k}^{\mu_{k} / \lambda_{k}} \leq \sum_{k=1}^{r} \frac{\mu_{k}}{\lambda_{k}} a_{k}, \quad a_{k} \geq 0
$$

to obtain

$$
\begin{aligned}
\prod_{k=1}^{r}\left|u_{k}(x, y)\right|^{\mu_{k}} & \leq \prod_{k=1}^{r} q\left(x, y, \lambda_{k}, \mu_{k}\right)\left(\int_{E} \int_{E^{\prime}}\left|D^{2 n} u_{k}(s, t)\right|^{\lambda_{k}} d s d t\right)^{\mu_{k} / \lambda_{k}} \\
& \leq \prod_{k=1}^{r} q\left(x, y, \lambda_{k}, \mu_{k}\right) \sum_{k=1}^{r} \frac{\mu_{k}}{\lambda_{k}} \int_{E} \int_{E^{\prime}}\left|D^{2 n} u_{k}(s, t)\right|^{\lambda_{k}} d s d t .
\end{aligned}
$$

Now multiplying both sides of (2.24) by $p(x, y)$ and then integrating over $E \times E^{\prime}$, we obtain (2.22).

Corollary 2.5. Let the conditions of Theorem 2.4 be satisfied. Then the following inequality holds

$$
\int_{E} \int_{E^{\prime}} p(x, y) \prod_{k=1}^{r}\left|u_{k}(x, y)\right|^{\mu_{k}} d x d y<K_{1}^{\prime} \int_{E} \int_{E^{\prime}} p(x, y) d x d y \sum_{k=1}^{r} \frac{\mu_{k}}{\lambda_{k}} \int_{E} \int_{E^{\prime}}\left|D^{2 n} u_{k}(x, y)\right|^{\lambda_{k}} d x d y,
$$

where

$$
K_{1}^{\prime}=\left(\frac{1}{2^{n+1}}\right)^{\sum_{k=1}^{r} \mu_{k}} \prod_{i=1}^{n}\left[\left(b_{i}-a_{i}\right)\left(b_{i}-a_{i}\right)\right]^{-1+\sum_{k=1}^{r} \mu_{k}}
$$

This is just a general form of the following inequality which was established by Agarwal and Sheng [15]:

$$
\int_{E} p(x) \prod_{k=1}^{r}\left|u_{k}(x)\right|^{\mu_{k}} d x<K_{1} \int_{E} p(x) d x \sum_{k=1}^{r} \frac{\mu_{k}}{\lambda_{k}} \int_{E}\left|D^{n} u_{k}(x)\right|^{\lambda_{k}} d x,
$$

where

$$
K_{1}=\left(\frac{1}{2^{n}}\right)^{\sum_{k=1}^{r} \mu_{k}} \prod_{i=1}^{n}\left(b_{i}-a_{i}\right)^{-1+\sum_{k=1}^{r} \mu_{k}}
$$

Remark 2.6. For $p(x, y)=1$, the inequality (2.22) becomes

$$
\int_{E} \int_{E^{\prime}} \prod_{k=1}^{r}\left|u_{k}(x, y)\right|^{\mu_{k}} d x d y \leq K_{2}^{\prime} \sum_{k=1}^{r} \frac{\mu_{k}}{\lambda_{k}} \int_{E} \int_{E^{\prime}}\left|D^{2 n} u_{k}(x, y)\right|^{\lambda_{k}} d x d y,
$$

where

$$
K_{2}^{\prime}=\left(\frac{1}{2} B^{2}\left(\frac{1+\sum_{k=1}^{r} \mu_{k}}{2}, \frac{1+\sum_{k=1}^{r} \mu_{k}}{2}\right)\right)^{n} \prod_{i=1}^{n}\left[\left(b_{i}-a_{i}\right)\left(d_{i}-c_{i}\right)\right]^{\sum_{k=1}^{r} \mu_{k}} .
$$

For $u(x, y)=u(x)$, the inequality (2.29) has been obtained by Agarwal and Sheng [15]. 
Theorem 2.7. Let $\lambda$ and $u(x, y)$ be as in Theorem 2.1, $\mu \geq 1$ be a given real number. Then the following inequality holds

$$
\int_{E} \int_{E^{\prime}}|u(x, y)|^{\lambda} d x d y \leq K_{3}^{\prime}(\lambda, \mu) \int_{E} \int_{E^{\prime}}\|\operatorname{grad} u(x, y)\|_{\mu}^{\lambda} d x d y
$$

where

$$
\begin{gathered}
K_{3}^{\prime}(\lambda, \mu)=\frac{1}{2 n} B^{2}\left(\frac{\lambda+1}{2}, \frac{\lambda+1}{2}\right) K\left(\frac{\lambda}{\mu}\right) \prod_{i=1}^{n}\left[\left(b_{i}-a_{i}\right)\left(d_{i}-c_{i}\right)\right]^{\lambda / n}, \\
\|\operatorname{grad} u(x, y)\|_{\mu}=\left(\sum_{i=1}^{n}\left|\frac{\partial^{2}}{\partial x_{i} \partial y_{i}} u(x, y)\right|^{\mu}\right)^{1 / \mu},
\end{gathered}
$$

and where $K(\lambda / \mu)=1$ if $\lambda \geq \mu$, and $K(\lambda / \mu)=n^{1-\lambda / \mu}$ if $0 \leq \lambda / \mu \leq 1$.

Proof. For each fixed $i, 1 \leq i \leq n$, in view of

$$
\left.u(x, y)\right|_{x_{i}=a_{i}}=0,\left.\quad u(x, y)\right|_{y_{i}=c_{i}}=0,\left.\quad u(x, y)\right|_{x_{i}=b_{i}}=0,\left.\quad u(x, y)\right|_{y_{i}=d_{i}}=0, \quad(i=1, \ldots, n),
$$

we have

$$
\begin{aligned}
& u(x, y)=\int_{a_{i}}^{x_{i}} \int_{c_{i}}^{y_{i}} \frac{\partial^{2}}{\partial s_{i} \partial t_{i}} u\left(x, y ; s_{i}, t_{i}\right) d s_{i} d t_{i} \\
& u(x, y)=\int_{x_{i}}^{b_{i}} \int_{y_{i}}^{d_{i}} \frac{\partial^{2}}{\partial s_{i} \partial t_{i}} u\left(x, y ; s_{i}, t_{i}\right) d s_{i} d t_{i}
\end{aligned}
$$

where

$$
u\left(x, y ; s_{i}, t_{i}\right)=u\left(x_{1}, \ldots, x_{i-1}, s_{i}, x_{i+1}, \ldots, x_{n}, y_{1}, \ldots, y_{i-1}, t_{i}, y_{i+1}, \ldots, y_{n}\right) .
$$

Hence from Hölder inequality with indices $\lambda$ and $\lambda /(1-\lambda)$, it follows that

$$
\begin{aligned}
& |u(x, y)|^{\lambda} \leq\left[\left(x_{i}-a_{i}\right)\left(y_{i}-d_{i}\right)\right]^{\lambda-1} \int_{a_{i}}^{x_{i}} \int_{c_{i}}^{y_{i}}\left|\frac{\partial^{2}}{\partial s_{i} \partial t_{i}} u\left(x, y ; s_{i}, t_{i}\right)\right|^{\lambda} d s_{i} d t_{i}, \\
& |u(x, y)|^{\lambda} \leq\left[\left(b_{i}-x_{i}\right)\left(d_{i}-y_{i}\right)\right]^{\lambda-1} \int_{x_{i}}^{b_{i}} \int_{y_{i}}^{d_{i}}\left|\frac{\partial^{2}}{\partial s_{i} \partial t_{i}} u\left(x, y ; s_{i}, t_{i}\right)\right|^{\lambda} d s_{i} d t_{i} .
\end{aligned}
$$

Multiplying (2.36), and then applying the Arithmetic-Geometric means inequality, to obtain

$$
|u(x, y)|^{\lambda} \leq \frac{1}{2}\left[\left(x_{i}-a_{i}\right)\left(y_{i}-c_{i}\right)\left(b_{i}-x_{i}\right)\left(d_{i}-y_{i}\right)\right]^{(\lambda-1) / 2} \times \int_{a_{i}}^{b_{i}} \int_{c_{i}}^{d_{i}}\left|\frac{\partial^{2}}{\partial s_{i} \partial t_{i}} u\left(x, y ; s_{i}, t_{i}\right)\right|^{\lambda} d s_{i} d t_{i},
$$

and now integrating (2.37) on $E \times E^{\prime}$, we arrive at

$$
\begin{aligned}
\int_{E} \int_{E^{\prime}}|u(x, y)|^{\lambda} d x d y \leq & \int_{a_{i}}^{b_{i}} \int_{\mathcal{c}_{i}}^{d_{i}} \frac{1}{2}\left[\left(x_{i}-a_{i}\right)\left(y_{i}-c_{i}\right)\left(b_{i}-x_{i}\right)\left(d_{i}-y_{i}\right)\right]^{(\lambda-1) / 2} d x_{i} d y_{i} \\
& \times \int_{E} \int_{E^{\prime}}\left|\frac{\partial^{2}}{\partial x_{i} \partial y_{i}} u(x, y)\right|^{\lambda} d x d y .
\end{aligned}
$$


Next, multiplying the inequality (2.38) for $1 \leq i \leq n$, and using the Arithmetic-Geometric means inequality, and in view of the following inequality:

$$
\sum_{i=1}^{n} a_{i}^{\alpha} \leq K(\alpha)\left(\sum_{i=1}^{n} a_{i}\right)^{\alpha}, \quad a_{i}>0,
$$

where $K(\alpha)=1$ if $\alpha \geq 1$, and $K(\alpha)=n^{1-\alpha}$ if $0 \leq \alpha \leq 1$, we get

$$
\begin{aligned}
\int_{E} \int_{E^{\prime}}|u(x, y)|^{\lambda} d x d y \leq & \prod_{i=1}^{n}\left(\int_{a_{i}}^{b_{i}} \int_{c_{i}}^{d_{i}} \frac{1}{2}\left[\left(x_{i}-a_{i}\right)\left(y_{i}-c_{i}\right)\left(b_{i}-x_{i}\right)\left(d_{i}-y_{i}\right)\right]^{(\lambda-1) / 2} d x_{i} d y_{i}\right)^{1 / n} \\
& \times \prod_{i=1}^{n}\left(\int_{E} \int_{E^{\prime}}\left|\frac{\partial^{2}}{\partial x_{i} \partial y_{i}} u(x, y)\right|^{\lambda} d x d y\right)^{1 / n} \\
\leq & \frac{1}{2 n} \prod_{i=1}^{n}\left(\int_{a_{i}}^{b_{i}} \int_{c_{i}}^{d_{i}} \frac{1}{2}\left[\left(x_{i}-a_{i}\right)\left(y_{i}-c_{i}\right)\left(b_{i}-x_{i}\right)\left(d_{i}-y_{i}\right)\right]^{(\lambda-1) / 2} d x_{i} d y_{i}\right)^{1 / n} \\
& \times \sum_{i=1}^{n} \int_{E} \int_{E^{\prime}}\left|\frac{\partial^{2}}{\partial x_{i} \partial y_{i}} u(x, y)\right|^{\lambda} d x d y \\
\leq & \frac{1}{2 n} B^{2}\left(\frac{\lambda+1}{2}, \frac{\lambda+1}{2}\right) \prod_{i=1}^{n}\left[\left(b_{i}-a_{i}\right)\left(d_{i}-c_{i}\right)\right]^{\lambda / n} \\
& \times \int_{E} \int_{E^{\prime}}\|\operatorname{grad} u(x, y)\|_{\lambda}^{\lambda} d x d y, \\
\leq & K_{3}^{\prime}(\lambda, \mu) \int_{E} \int_{E^{\prime}}\|\operatorname{grad} u(x, y)\|_{\mu}^{\lambda} d x d y,
\end{aligned}
$$

where

$$
\begin{gathered}
K_{3}^{\prime}(\lambda, \mu)=\frac{1}{2 n} B^{2}\left(\frac{\lambda+1}{2}, \frac{\lambda+1}{2}\right) K\left(\frac{\lambda}{\mu}\right) \prod_{i=1}^{n}\left[\left(b_{i}-a_{i}\right)\left(d_{i}-c_{i}\right)\right]^{\lambda / n} \\
\|\operatorname{grad} u(x, y)\|_{\mu}=\left(\sum_{i=1}^{n}\left|\frac{\partial^{2}}{\partial x_{i} \partial y_{i}} u(x, y)\right|^{\mu}\right)^{1 / \mu}
\end{gathered}
$$

and where $K(\lambda / \mu)=1$ if $\lambda \geq \mu$, and $K(\lambda / \mu)=n^{1-\lambda / \mu}$ if $0 \leq \lambda / \mu \leq 1$.

Remark 2.8. Let $u(x, y)$ reduce to $u(x)$ in (2.31) and with suitable modifications, and let $\lambda \geq 2$, $\mu=2$, then (2.31) becomes

$$
\int_{E}|u(x)|^{\lambda} d x \leq K_{3}^{\prime}(\lambda, 2) \int_{E}\|\operatorname{grad} u(x)\|_{\mu}^{\lambda} d x .
$$

This is just a better inequality than the following inequality which was given by Pachpatte [17]

$$
\int_{E}|u(x)|^{\lambda} d x \leq \frac{1}{n}\left(\frac{\beta}{2}\right)^{\lambda} \int_{E}\|\operatorname{grad} u(x)\|_{\mu}^{\lambda} d x .
$$

Because for $\lambda \geq 2$, it is clear that $K_{3}^{\prime}(\lambda, 2)<(1 / n)(\beta / 2)^{\lambda}$, where $\beta=\max _{1 \leq i \leq n}\left(b_{i}-a_{i}\right)$. 
On the other hand, taking for $\mu=2, \lambda=2$ or $\mu=2, \lambda=4$ in (2.31) and let $u(x, y)$ reduce to $u(x)$ with suitable modifications, it follows the following Poincare-type inequalities:

$$
\begin{aligned}
& \int_{E}|u(x)|^{2} d x \leq \frac{\pi}{16 n} \beta^{2} \int_{E}\|\operatorname{grad} u(x)\|_{2}^{2} d x, \\
& \int_{E}|u(x)|^{4} d x \leq \frac{3 \pi}{256 n} \beta^{4} \int_{E}\|\operatorname{grad} u(x)\|_{2}^{4} d x .
\end{aligned}
$$

The inequalities (2.44) have been discussed in [18] with the right-hand sides, respectively, multiplied by $4 / \pi$ and $16 / 3 \pi$. Hence inequalities (2.44) are more strong results on these types of inequalities.

If $\mu \geq \lambda$, in the right sides of (2.31) we can apply Hölder inequality with indices $\mu / \lambda$ and $\mu /(\mu-\lambda)$, to obtain the following corollary.

Corollary 2.9. Let the conditions of Theorem 2.7 be satisfied and $\mu \geq \lambda$. Then

$$
\int_{E} \int_{E^{\prime}}|u(x, y)|^{\lambda} d x d y \leq K_{4}^{\prime}(\lambda, \mu)\left(\int_{E} \int_{E^{\prime}}\|\operatorname{grad} u(x, y)\|_{\mu}^{\mu} d x d y\right)^{\lambda / \mu},
$$

where

$$
K_{4}^{\prime}(\lambda, \mu)=K_{3}^{\prime}(\lambda, \mu) \prod_{i=1}^{n}\left[\left(b_{i}-a_{i}\right)\left(d_{i}-c_{i}\right)\right]^{(\mu-\lambda) / \mu} .
$$

Remark 2.10. Taking $u(x, y)=u(x)$ and with suitable modifications, the inequality (2.45) reduces to the following result which was given by Agarwal and Sheng [15]:

$$
\int_{E}|u(x)|^{\lambda} d x \leq K_{6}(\lambda, \mu)\left(\int_{E}\|\operatorname{grad} u(x)\|_{\mu}^{\mu} d x\right)^{\lambda / \mu}
$$

where

$$
\begin{aligned}
& K_{6}(\lambda, \mu)=K_{5}(\lambda, \mu) \prod_{i=1}^{n}\left(b_{i}-a_{i}\right)^{(\mu-\lambda) / \mu}, \\
& K_{5}(\lambda, \mu)=\frac{1}{2 n} B\left(\frac{1+\lambda}{2}, \frac{1+\lambda}{2}\right) K\left(\frac{\lambda}{\mu}\right) \prod_{i=1}^{n}\left(b_{i}-a_{i}\right)^{\lambda / n},
\end{aligned}
$$

and $K(\lambda / \mu)$ is as in Theorem 2.7 .

Taking $\lambda=1, \mu=2$ the inequality (2.45), (2.45) reduces to

$$
\left(\int_{E} \int_{E^{\prime}}|u(x, y)| d x d y\right)^{2} \leq K_{4}^{\prime}(1,2) \int_{E} \int_{E^{\prime}}\|\operatorname{grad} u(x, y)\|_{2}^{2} d x d y .
$$

This is just a general form of the following inequality which was given by Agarwal and Sheng [15].

$$
\left(\int_{E}|u(x)| d x\right)^{2} \leq\left[K_{6}(1,2)\right]^{2} \int_{E}\|\operatorname{grad} u(x)\|_{2}^{2} d x d y .
$$

Similar to the proof of Theorem 2.7, we have the following theorem. 
Theorem 2.11. For $u_{k}(x, y) \in G\left(E \times E^{\prime}\right), \mu_{k} \geq 1,1 \leq k \leq r$. Then the following inequality holds

$$
\int_{E} \int_{E^{\prime}}\left(\prod_{i=1}^{n}\left|u_{k}(x, y)\right|^{\mu_{k}}\right)^{1 / r} d x d y \leq K_{5}^{\prime} \int_{E} \int_{E^{\prime}} \sum_{k=1}^{r}\left\|\operatorname{grad} u_{k}(x, y)\right\|_{\mu_{k}}^{\mu_{k}} d x d y,
$$

where

$$
K_{5}^{\prime}=\frac{1}{2 n r} B^{2}\left(\frac{1+(1 / r) \sum_{k=1}^{r} \mu_{k}}{2}, \frac{1+(1 / r) \sum_{k=1}^{r} \mu_{k}}{2}\right) \prod_{i=1}^{n}\left[\left(b_{i}-a_{i}\right)\left(d_{i}-c_{i}\right)\right]^{\sum_{k=1}^{r} \mu_{k} / n r} .
$$

Remark 2.12. Taking $u(x, y)=u(x)$ and with suitable modifications, the inequality (2.51) reduces to the following result:

$$
\int_{E}\left(\prod_{i=1}^{n}\left|u_{k}(x)\right|^{\mu_{k}}\right)^{1 / r} d x \leq K_{9} \int_{E} \sum_{k=1}^{r}\|\operatorname{grad} u(x)\|_{\mu_{k}}^{\mu_{k}} d x
$$

where

$$
K_{9}=\frac{1}{2 n r} B\left(\frac{1+(1 / r) \sum_{k=1}^{r} \mu_{k}}{2}, \frac{1+(1 / r) \sum_{k=1}^{r} \mu_{k}}{2}\right) \prod_{i=1}^{n}\left(b_{i}-a_{i}\right)^{\sum_{k=1}^{r} \mu_{k} / n r} .
$$

In [19], Pachpatte proved the inequality (2.53) for $\mu_{k} \geq 2,1 \leq k \leq r$ with $K_{9}$ replaced by $(1 / n r)(\beta / 2)^{\sum_{k=1}^{r} \mu_{k} / r}$, where $\beta$ is as in Remark 2.8. It is clear that $K_{9}<(1 / n r)(\beta / 2)^{\sum_{k=1}^{r} \mu_{k} / r}$, and hence (2.53) is a better inequality than a result of Pachpatte.

Similarly, all other results in [15] also can be generalized by the same way. Here, we omit the details.

\section{Acknowledgments}

Research is supported by Zhejiang Provincial Natural Science Foundation of China(Y605065), Foundation of the Education Department of Zhejiang Province of China (20050392). Research is partially supported by the Research Grants Council of the Hong Kong SAR, China (Project No.:HKU7016/07P).

\section{References}

[1] R. P. Agarwal and P. Y. H. Pang, Opial Inequalities with Applications in Differential and Difference Equations, vol. 320 of Mathematics and Its Applications, Kluwer Academic Publishers, Dordrecht, The Netherlands, 1995.

[2] R. P. Agarwal and V. Lakshmikantham, Uniqueness and Nonuniqueness Criteria for Ordinary Differential Equations, vol. 6 of Series in Real Analysis, World Scientific, Singapore, 1993.

[3] R. P. Agarwal and E. Thandapani, "On some new integro-differential inequalities," Analele Ştiinţifice ale Universităţii "Al. I. Cuza" din Iaşi, vol. 28, no. 1, pp. 123-126, 1982.

[4] D. Baĭnov and P. Simeonov, Integral Inequalities and Applications, vol. 57 of Mathematics and Its Applications, Kluwer Academic Publishers, Dordrecht, The Netherlands, 1992.

[5] J. D. Li, "Opial-type integral inequalities involving several higher order derivatives," Journal of Mathematical Analysis and Applications, vol. 167, no. 1, pp. 98-110, 1992.

[6] D. S. Mitrinovič, J. E. Pečarić, and A. M. Fink, Inequalities Involving Functions and Their Integrals ang Derivatives, Kluwer Academic Publishers, Dordrecht, The Netherlands, 1991. 
[7] W.-S. Cheung, "On Opial-type inequalities in two variables," Aequationes Mathematicae, vol. 38, no. 2-3, pp. 236-244, 1989.

[8] W.-S. Cheung, "Some new Opial-type inequalities," Mathematika, vol. 37, no. 1, pp. 136-142, 1990.

[9] W.-S. Cheung, "Some generalized Opial-type inequalities," Journal of Mathematical Analysis and Applications, vol. 162, no. 2, pp. 317-321, 1991.

[10] W.-S. Cheung, "Opial-type inequalities with $m$ functions in $n$ variables," Mathematika, vol. 39, no. 2, pp. 319-326, 1992.

[11] P. S. Crooke, "On two inequalities of Sobolev type," Applicable Analysis, vol. 3, no. 4, pp. 345-358, 1974.

[12] B. G. Pachpatte, "Opial type inequality in several variables," Tamkang Journal of Mathematics, vol. 22, no. 1, pp. 7-11, 1991.

[13] B. G. Pachpatte, "On some new integral inequalities in two independent variables," Journal of Mathematical Analysis and Applications, vol. 129, no. 2, pp. 375-382, 1988.

[14] X.-J. Wang, "Sharp constant in a Sobolev inequality," Nonlinear Analysis: Theory, Methods \& Application, vol. 20, no. 3, pp. 261-268, 1993.

[15] R. P. Agarwal and Q. Sheng, "Sharp integral inequalities in $n$ independent variables," Nonlinear Analysis: Theory, Methods \& Applications, vol. 26, no. 2, pp. 179-210, 1996.

[16] P. D. Smith and E. W. Stredulinsky, "Nonlinear elliptic systems with certain unbounded coefficients," Communications on Pure and Applied Mathematics, vol. 37, no. 4, pp. 495-510, 1984.

[17] B. G. Pachpatte, "A note on Poincaré and Sobolev type integral inequalities," Tamkang Journal of Mathematics, vol. 18, no. 1, pp. 1-7, 1987.

[18] B. G. Pachpatte, "On multidimensional integral inequalities involving three functions," Soochow Journal of Mathematics, vol. 12, pp. 67-78, 1986.

[19] B. G. Pachpatte, "On Sobolev type integral inequalities," Proceedings of the Royal Society of Edinburgh A, vol. 103, no. 1-2, pp. 1-14, 1986. 